chanter tandis que la lune se levait sur le lac Couchiching, de plusieurs promenades, à Woods Hole ou le long de la plage de Sylt, où le battement du ressac rythmait les discussions.

Il y aurait tant à dire, mais j'aimerais ici souligner un point pour lequel le rôle de Klaus Hasselmann a été déterminant et dont nous étions nombreux à avoir conscience : la construction d'un espace de projet européen. En Norvège, en Italie, en France, en Espagne, peut-être moins au Royaume-Uni, en Allemagne ou aux Pays-Bas, nous avions travaillé jusque-là dans des équipes petites et isolées. Le souffle de collaboration européenne que Klaus Hasselmann nous a communiqué dans l'application concrète de ses travaux pour la prévision opérationnelle des vagues océaniques a été déterminant. Il a fait de l'Europe le centre mondial de la modélisation des vagues et permis d'exporter le modèle WAM dans le monde entier. Qu'il en soit ici chaleureusement remercié.

\section{Anne Guillaume}

\footnotetext{
Pour en savoir plus :

Allender J.H., Barnett T.P., Bertotti L., Bruinsma J., Cardone V.J., Cavaleri L., Ephraums J., Golding B., Greenwood A., Guddal J., Günther H., Hasselmann K., Hasselmann S., Joseph P., Kawai S., Komen G.J., Lawson L., Linné H., Long R.B., Lybanon M., Maeland E., Rosenthal W., Toba Y., Uji T., de Voogt W.J.P., 1985. Ocean Wave Modeling, Part 1: The Sea Wave Modelling Project (SWAMP), Principal results and conclusions. Plenum Publishing Corporation.

Bauer E., Bertotti L., Cardone C.V., Ewing J.A., Greenwood J.A., Guillaume A., Hasselmann K., Hasselmann S., Janssen P.A.E.M., Komen G.J., Lionello P., Reistad M., Zambresky L., 1988. The WAM Model - A third generation ocean wave prediction model. J. Phys. Oceanogr., 18, 1775-1810.

Eckert M., 2017. Ludwig Prandtl and the growth of fluid mechanics in Germany. Comptes Rendus Mécanique, 345, 467-476. doi: 10.1016/j.crme. 2017.05.005

Hasselmann K., 1960. Grundgleichungen der Seegangsvoraussage. Schiffstechnik, 7, S. 191-195.

Hasselmann K., 1961. Über den nichtlinearen Energieaustausch innerhalb eines Seegangsspektrums. Sonderdruck aus Zeitschrift für Angewandte Mathematik und Mechanik, Sonderheft (GAMM-Tagung Würzburg), 41.

Hasselmann K., Barnett T.P., Bouws E., Carlson H., Cartwright D.E., Enke K., Ewing J.A., Gienapp H., Hasselmann D.E., Kruseman P., Meerburg A., Müller P., Olbers D.J., Richter K., Sell W., Walden H., 1973. Measurements of wind-wave growth and swell decay during the Joint North Sea Wave Project (JONSWAP). Ergänzungsheft zur Deutschen Hydrographischen Zeitschrift, Reihe A, 12.

Hasselmann K., Ross D.B., Müller P., Sell W., 1976. A parametric wave prediction model. J. Phys. Oceanogr., 6, 200-228.

Hasselmann S., Hasselmann K., 1985. Computations and parameterizations of the nonlinear energy transfer in a gravity wave spectrum. Part I: A new method for efficient computations of the exact nonlinear transfer integral. J. Phys. Oceanogr., 15, 1369-1377.

Snodgrass F.E., Groves G.W., Hasselmann K.F., Miller C.R., Munk W.H., Powers W.H., 1966. Propagation of ocean swell across the Pacific. Phil. Trans. R. Soc. A, 1103, 259, 431-497.
}

\title{
Le PMIP fête ses 30 ans
}

Depuis 30 ans, le Paleoclimate Modeling Intercomparison Project (PMIP) mobilise les groupes de modélisation et les spécialistes des reconstructions paléoclimatiques avec l'ambition de comprendre les variations climatiques passées et tester la capacité des modèles de climat à représenter un climat différent de l'actuel. Ce projet a été lancé en 1991 au cours d'un colloque international organisé en France par Jean Jouzel et Sylvie Joussaume. Cette mise en perspective à long terme reste toujours d'actualité au moment où le dernier rapport du Giec fait ressortir qu'il faut remonter plus de 2 millions d'années dans le passé pour retrouver les taux actuels de $\mathrm{CO}_{2}$ dans l'atmosphère, et que l'augmentation de la température moyenne du globe depuis la période préindustrielle excède les variations des 100000 dernières années (IPCC, 2021).

La spécificité du PMIP est de tester en conditions paléoclimatiques les modèles utilisés pour les projections futures. Ces modèles ont évolué à partir de modèles d'atmosphère vers des modèles complets du système Terre (figure 1). Néanmoins, la complexité

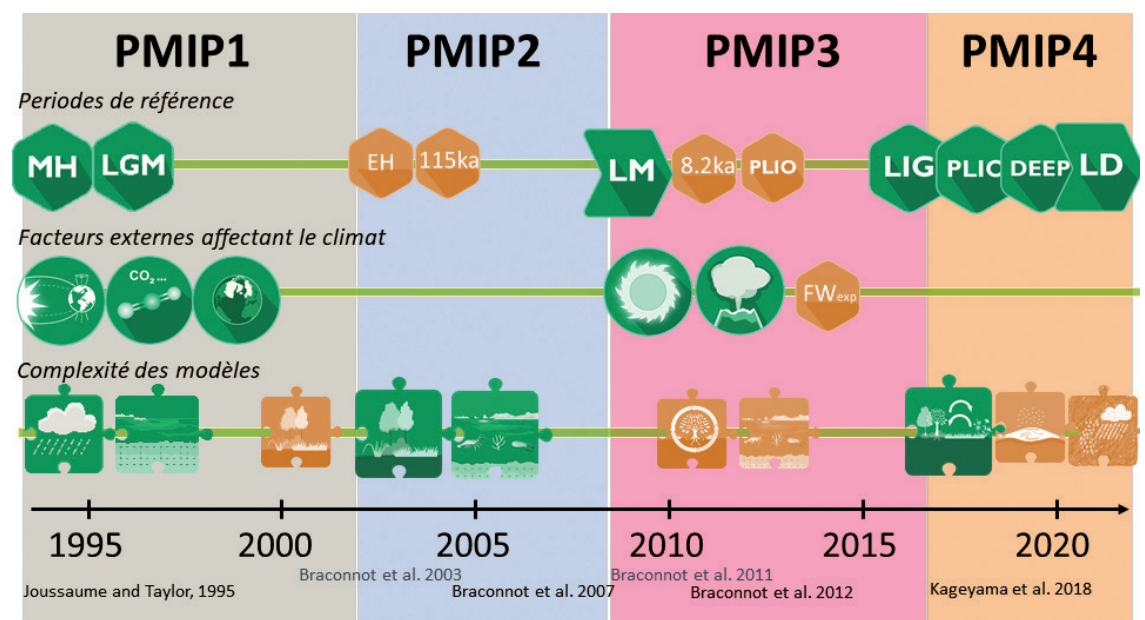

Figure 1. Frise montrant l'évolution du PMIP, en mettant l'accent sur les quatre phases, les périodes de référence considérées, les facteurs externes affectant le climat et la complexité des modèles. Les éléments en vert correspondent à ce qui est traité par l'ensemble des groupes participant au projet, alors que les éléments en orange indiquent des facteurs additionnels considérés par un sousensemble de participants. Les références sont les références clés des quatre phases, en noir dans des revues à comité de lecture et en bleu dans le magazine du programme Clivar du Programme mondial de recherche sur le climat (PMRC).

des modèles et le niveau d'intégration du cycle du carbone, de la dynamique de la végétation ou des aérosols varient encore fortement d'un modèle à l'autre. À chaque nouvelle phase du PMIP (figure 1), les ordinateurs crépitent dans plus de 20 pays répartis sur le continent américain, Europe et Asie, et les bases de données absorbent des pétaoctects bien formatés pour favoriser les échanges, ainsi que les comparaisons modèles-modèles et modèles-données. Sédiments marins, carottes de glace, pollen, niveaux passés des lacs, cernes 

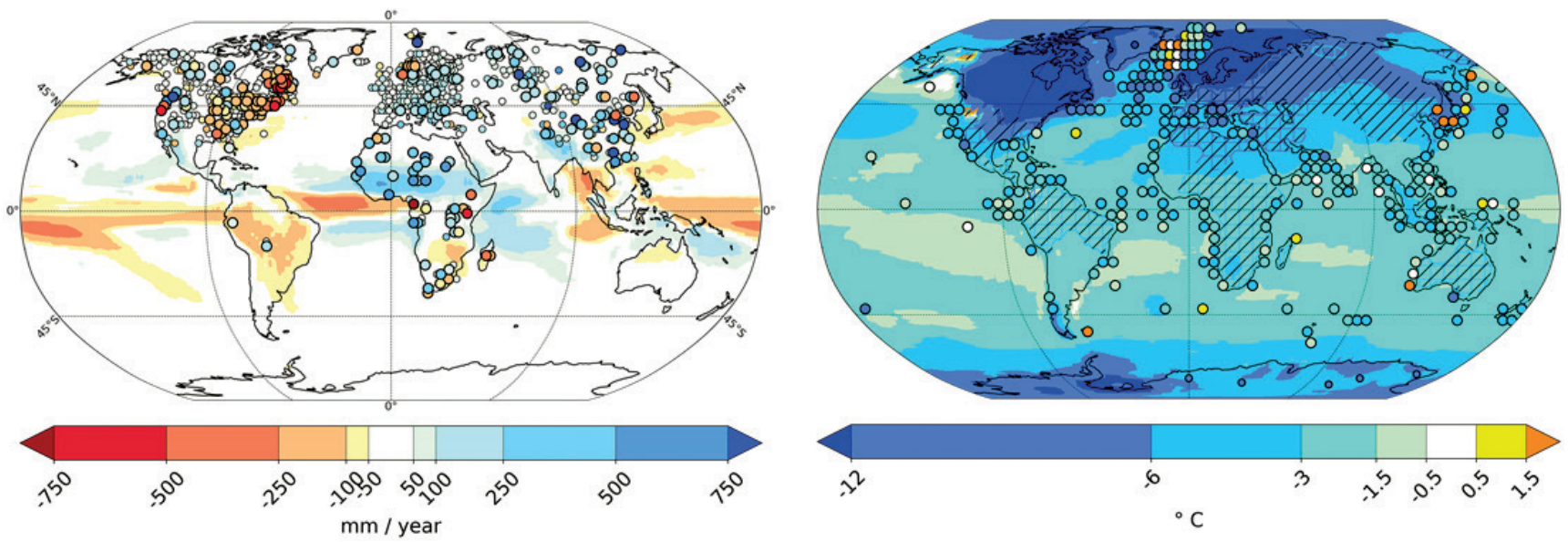

Figure 2. Changements annuels de précipitations simulées pour l'Holocène moyen (il y a 6000 ans, à gauche) et de température de surface pour le dernier maximum glaciaire (il y a 21000 ans, à droite). Le fond de carte représente la moyenne multi-modèles calculée à partir des résultats de PMIP4. L'échelle de couleur est irrégulière pour mieux caractériser les différences. Les ronds colorés sont issus des reconstructions et indiqués aux sites de prélèvement. Pour le dernier maximum glaciaire, de nouvelles reconstructions couvrent des régions plus larges et ont été reprojetées sur une grille. Les zones couvertes sont indiquées par des hachures. Lorsque les données ne sont pas dans la gamme des modèles, les valeurs ressortent dans une couleur différente du fond de carte sur la grille des données.

d'arbres, spéléothèmes, coraux ou coquilles sont tous réquisitionnés, analysés et rassemblés pour fournir des reconstructions climatiques pour les périodes clefs du projet.

Au début des années 1990, le passage d'initiatives de groupes ou de projets individuels à une entreprise internationale s'est fait grâce à la rencontre de deux approches. Du côté des paléoclimats, le groupe COHMAP (Cooperative Holocene Mapping Project, 1988) a démontré l'intérêt de la mise en regard systématique des résultats des simulations climatiques et des reconstructions paléoclimatiques réalisées à partir des différents types d'indicateurs marins ou terrestres. Du côté des climats actuel et futur, le projet AMIP s'est mis en place pour offrir un cadre de comparaison systématique des résultats des modèles d'atmosphère (Gates, 1992). Ces réflexions ont conduit à choisir le climat du dernier maximum glaciaire, il y a 21000 ans, et de l'Holocène moyen, il y a 6000 ans, comme périodes de référence pour PMIP1 (figures 1 et 2). Sous la houlette de Sylvie Joussaume (France) et de Karl Taylor (États-Unis), le PMIP a été dès le départ inséré dans une dynamique internationale en plein essor portée par le Programme mondial de recherche sur le climat (PMRC) et le projet Past Global Changes (PAGES).

Les résultats du PMIP ont apporté des éclairages sur des questions pour lesquelles nos connaissances restent incertaines. Ils ont alimenté les différents rapports du Giec. Le dernier maximum glaciaire remet par exemple régulièrement en question l'amplitude des changements de température reconstruite ou simulée et l'adéquation entre changement de température sur terre et sur mer. À cette époque, les calottes de glace de l'hémisphère Nord et une plus faible teneur en dioxyde de carbone dans l'atmosphère (180 ppm au lieu des 410 ppm actuels) sont à l'origine d'un climat froid. Malgré un bon accord entre climats simulé et reconstruit pour les grands changements de température de surface, le refroidissement continental simulé reste insuffisant en Méditerranée et dans les régions tropicales bien échantillonnées (figure 2). Plus proches de la période actuelle, des caractéristiques légèrement différentes, par rapport à aujourd'hui, de l'orbite terrestre autour du Soleil à l'Holocène moyen favorisent une amplitude accrue (réduite) de la saisonnalité dans l'hémisphère Nord (hémisphère Sud) et l'ensoleillement des hautes latitudes. Les simulations reproduisent l'accroissement des précipitations de mousson reconstruites dans les tropiques, mais ont encore tendance à sous-estimer les grandes variations de pluie au SahelSahara (figure 2).
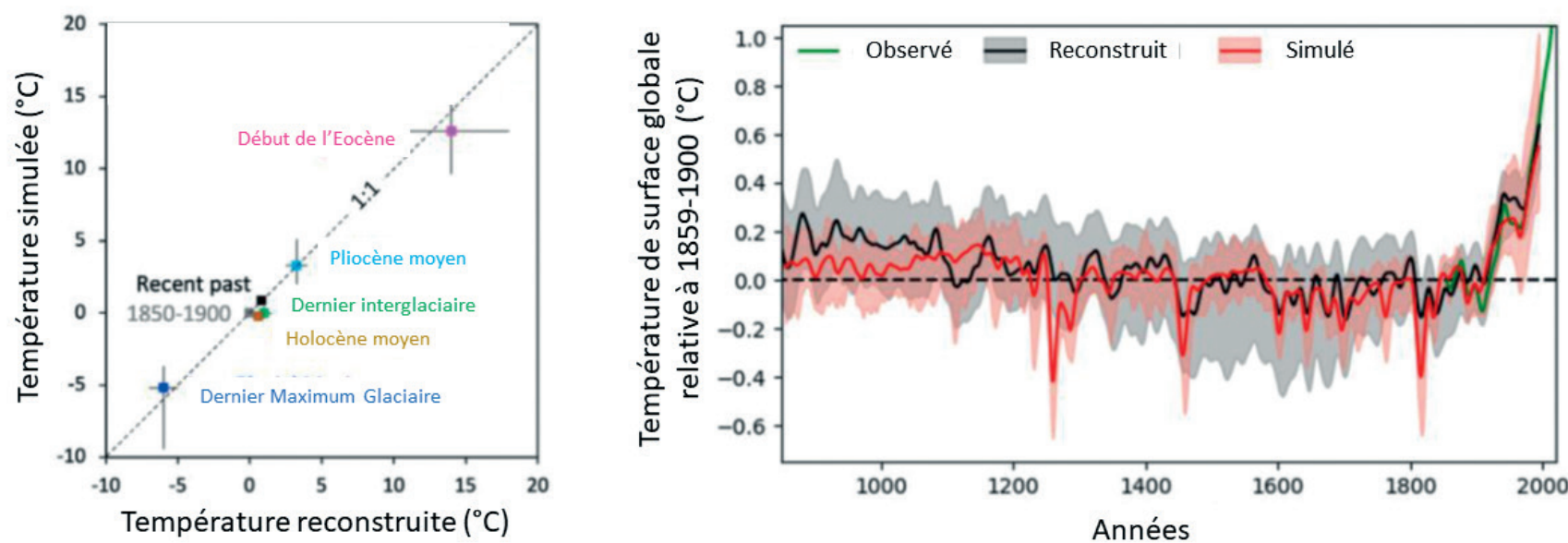

Figure 3. Figure adaptée du 6e rapport du groupe 1 du Giec (2021). La partie gauche montre les différences de température de surface globale par rapport à la période 1850-1900 pour différentes périodes paléoclimatiques, en faisant ressortir l'adéquation entre températures simulées et températures reconstruites à partir de différentes archives climatiques. La partie droite représente l'évolution de la température globale pour le dernier millénaire pour les reconstructions, les simulations et les observations pour la période récente. 
Au fil du temps, les périodes d'intérêt ont également été élargies au dernier millénaire, au Pliocène moyen (il y a 3,3 millions d'années) et au dernier interglaciaire (il y a 127000 ans). Ces périodes font aussi partie de l'ensemble CMIP6 de simulations des climats passés, présents et futurs (Eyring et al., 2016; Kageyama et al., 2018). La phase PMIP4 inclut, de plus, les climats plus anciens (deepMIP) et un grand défi autour de la dernière déglaciation (figure 1). Croiser les résultats des différentes périodes permet de vérifier la façon dont les modèles reproduisent les changements de températures suivant les perturbations apportées (figure 3). Les réflexions associées ont contribué à affiner la borne supérieure de cette sensibilité climatique (Sherwood et al., 2020). Les simulations du climat du dernier millénaire ont aussi montré que les variations de l'éclairement solaire avaient un poids très faible par rapport aux éruptions volcaniques pour expliquer les variations observées dans une période où les émissions de gaz à effet de serre par l'activité humaine sont faibles (figure 3 ).

Un accent croissant est mis sur la variabilité. Les résultats indiquent que les variations climatiques du dernier millénaire auraient été engendrées par la variabilité interne du climat (MassonDelmotte et al., 2013). Ils questionnent la capacité des modèles à représenter la variabilité climatique régionale aux échelles décennales et au-delà (Laepple et Huybers, 2014). Climats froids et chauds se caractérisent par une augmentation de variabilité, alors que la variabilité des précipitations est réduite en climat froid et augmentée en climat chaud (Rehfeld et al., 2020). Les simulations reflètent la réduction de l'amplitude des événements El Niño à l'Holocène moyen, mais

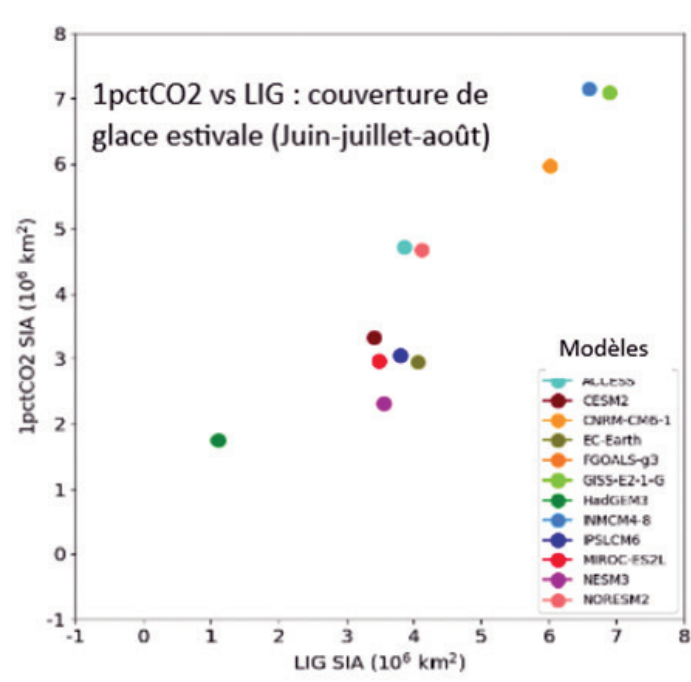

\section{Moyenne multimodèles}

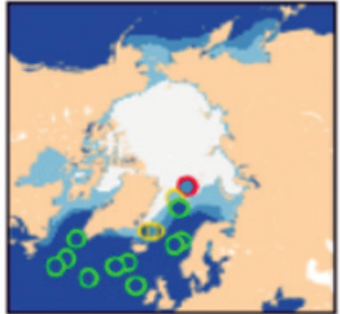

Nbre modèles en accord avec les reconstructions:

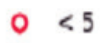

de 5 à 9

o $>9$

Concentration de glace \%

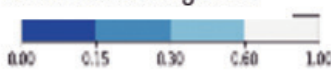

Figure 4. Couverture de glace de mer simulée par l'ensemble des modèles pour le dernier interglaciaire, il y a 127000 ans. Les données de couverture de glace estivale du dernier interglaciaire sont comparées pour chaque modèle avec les résultats des simulations où la concentration en dioxyde de carbone est augmentée de $1 \%$ par an dans l'atmosphère. La carte représente la moyenne d'ensemble des simulations et les ronds de couleur le nombre de modèles en accord avec les données des sites de prélèvement.

la sous-estiment (Emile-Geay et al., 2016). Pour les autres périodes, il y a peu d'accord entre les modèles, tout comme pour le climat futur (Brown et al., 2020).

Des comparaisons modèles-données plus fines à l'échelle régionale ouvrent la voie à une évaluation directe de certains aspects des projections climatiques futures. Par exemple, la diminution du minimum de couverture de glace estivale simulé pour le dernier interglaciaire est liée à la diminution du minimum de couverture de glace simulé pour le futur (figure 4). Les reconstructions de couverture de glace indiquent les régions où les simulations sont en accord avec les données et les zones d'incertitudes. De plus, la compréhension des différentes rétroactions liées au changement de couverture de glace, de la vapeur d'eau ou des nuages offre une meilleure caractérisation des facteurs locaux intervenant dans la sensibilité climatique.

Le PMIP continue d'attirer de nouvelles idées et de jeunes chercheurs. Une évolution vers une approche dynamique des changements climatiques permettant de mieux éclairer les interactions entre rétroactions lentes et rapides est en train de se dessiner pour les futures explorations.

Pascale Braconnot, Masa Kageyama, Jean-Yves Peterschmitt

Laboratoire des sciences du climat et de l'environnement, Institut Pierre-Simon Laplace

Catherine Michaut

Institut Pierre-Simon Laplace

Brown J.R. et al., 2020. Comparison of past and future simulations of ENSO in CMIP5/PMIP3 and CMIP6/PMIP4 models. Clim. Past, 16, 1777-1805. COHMAP members, 1988. Climatic changes of the last 18,000 years: observations and model simulations. Science, 241, 1043-1052.

Emile-Geay J. et al., 2016. Links between tropical Pacific seasonal, interannual and orbital variability during the Holocene. Nat. Geosci., 9, 168-173. Eyring V., Bony S., Meehl G.A., Senior C.A., Stevens B., Stouffer R.J., Taylor K.E., 2016. Overview of the Coupled Model Intercomparison Project Phase 6 (CMIP6) experimental design and organization. Geosci. Model Dev., 9, 1937-1958.

Gates W.L., 1992. AMIP: the Atmospheric Model Intercomparison Project. Bull. Amer. Meteorol. Soc., 73, 1962-1970.

IPCC, 2021. Climate Change 2021: The Physical Science Basis. Contribution of Working Group I to the Sixth Assessment Report of the Intergovernmental Panel on Climate Change [Masson-Delmotte V., Zhai P., Pirani A., Connors S.L., Péan C., Berger S., Caud N., Chen Y., Goldfarb L., Gomis M.I., Huang M., Leitzell K., Lonnoy E., Matthews J.B.R., Maycock T.K., Waterfield T., Yelekçi O., Yu R., Zhou B. (Eds.)]. Cambridge University Press. In Press.

Kageyama M. et al., 2018. The PMIP4 contribution to CMIP6-Part 1: Overview and over-arching analysis plan. Geosci. Model Dev., 11, $1033-1057$.

Laepple T., Huybers P., 2014. Ocean surface temperature variability: Large model-data differences at decadal and longer periods. Proc. Nat. Acad. Sci., $111,16682-16687$.

Masson-Delmotte V., Schulz M., Abe-Ouchi A., Beer J., Ganopolski A., González Rouco J F., Jansen E., Lambeck K., Luterbacher J., Naish T., Osborn T., Otto-Bliesner B., Quinn T., Ramesh R., Rojas M., Shao X., Timmermann A., 2013. Information from Paleoclimate Archives. In: Climate Change 2013: The Physical Science Basis. Contribution of Working Group I to the Fifth Assessment Report of the Intergovernmental Panel on Climate Change, Stocker T.F., D. Qin, G.-K. Plattner, M. Tignor, S.K. Allen, J. Boschung, A. Nauels, Y. Xia, V. Bex and P.M. Midgley (Ed.), Cambridge University Press. Rehfeld K., Hébert R., Lora J.M., Lofverstrom M., Brierley C.M., 2020. Variability of surface climate in simulations of past and future. Earth Syst. Dyn., 11, 447-468.

Sherwood S.C. et al., 2020. An assessment of Earth's climate sensitivity using multiple lines of evidence. Rev. Geophys., 58, e2019RG000678. 


\section{météo}

PRIX ANDRÉ PRUD'HOMME APPEL À CANDIDATURES 2022

Un concours national pour les jeunes chercheurs

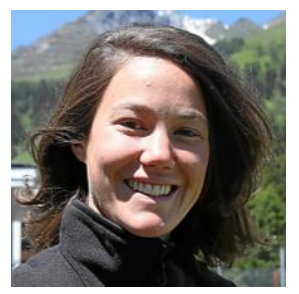

Neige Calonne Prix 2016

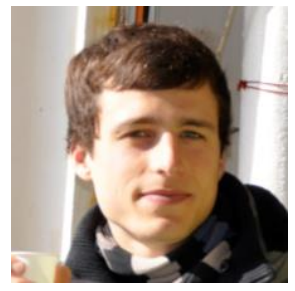

Casimir de Lavergne Prix 2017

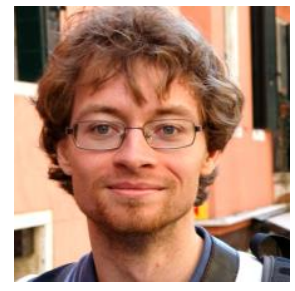

Alexandre Pohl Prix 2018

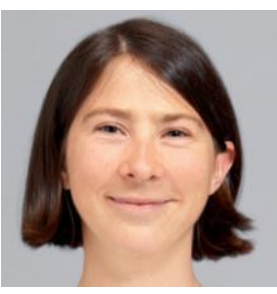

Fanny Brun Prix 2019

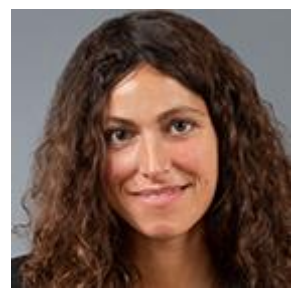

Lia Siegelman Prix 2020

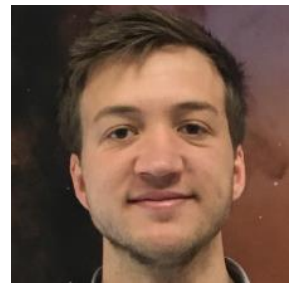

Alex Ayet Prix 2021

Le prix André Prud'homme s'adresse à des jeunes chercheurs ayant soutenu une thèse de doctorat dans le domaine des sciences de l'atmosphère et du climat.

Il a été créé par l'association Météo et Climat en 1992 afin d'honorer la mémoire d'André Prud'homme, ancien ingénieur à Météo-France.

\section{RĖGLEMENT ET MODALITÉS}

\section{Domaines de la thèse}

Météorologie - Physique et chimie de l'atmosphère - Paléoclimatologie - Climatologie (y compris les aspects océanographiques). Une thèse en planétologie n'est éligible que si elle a un lien explicite avec des processus concernant les systèmes atmosphériques ou océaniques terrestres.

\section{Lieu et année de soutenance de la thèse}

La thèse doit avoir été soutenue dans une université ou une école doctorale française en 2020 ou en 2021. Une candidature peut être présentée deux années consécutives.

\section{Engagement du(de la) lauréat(e)}

Le(a) lauréat(e) s'engage à publier un article présentant les points essentiels de ses travaux dans la revue scientifique La Météorologie, éditée par Météo et Climat www.lameteorologie.fr.

\section{COMMENT CONCOURIR AU PRIX 2022 ?}

Il suffit d'envoyer avant le $1^{\text {er }}$ février 2022

Par e-mail à info@meteoetclimat.fr :

- Votre lettre de candidature précisant le titre de votre thèse, lieu et année de soutenance, directeur de thèse ainsi que vos coordonnées postales, adresse e-mail et numéro de téléphone.

- Votre thèse en version électronique

- Le résumé de la thèse (une page $\mathrm{A} 4$ maximum).

- La liste de vos publications

- Les avis des rapporteurs de votre thèse

Par voie postale à Météo et Climat 73, avenue de Paris 94165 Saint-Mandé cedex:

- 1 exemplaire papier de votre thèse

\section{JURY}

L'évaluation des dossiers est effectuée par un jury présidé par Laurence EYMARD (directrice de recherche CNRS émérite) et composé de spécialistes des sciences de l'atmosphère et du climat.

\author{
ATTRIBUTION DU PRIX \\ La décision d'attribution du prix sera communiquée \\ au printemps 2022. \\ Le prix d'un montant de $1800 €$ sera remis en janvier \\ 2023, au cours d'une cérémonie à l'issue de laquelle \\ le(a) lauréat(e) fera un exposé sur sa thèse.
} + d'infos : Tél. : 0149571879 - info@meteoetclimat.fr - www.meteoetclimat.fr 


\section{PRIX PERRINDE BRICHAMBAUT APPEL À CANDIDATURES 2022}

\section{Un concours national sur la météo et le climat à destination des écoles, les collèges et les lycées}

Proposé chaque année par l'association Météo et Climat, le prix Perrin de Brichambaut a pour objectif de promouvoir et d'encourager l'action culturelle et scientifique des établissements scolaires dans les domaines des sciences de l'atmosphère et du climat. Il a été créé en 1997 afin d'honorer la mémoire de Christian Perrin de Brichambaut, ancien ingénieur à Météo-France et ancien président de Météo et Climat qui a beaucoup œuvré en faveur de la vulgarisation scientifique.

\section{RĖGLEMENT ET MODALITÉS}

Ce concours s'adresse aux écoles primaires, aux collèges et aux lycées qui réalisent au cours de l'année scolaire 2021-2022 un projet sur le thème de la météorologie, du climat et disciplines connexes explicitant un lien avec ces deux thématiques (développement durable, eau, environnement, énergie etc.).

Les établissements scolaires étrangers sont autorisés à concourir dans la mesure où le projet soumis et ses annexes sont rédigés en langue française.

\section{JURY ET CRITĖRES D'APPRÉCIATION}

Les projets sont évalués par un jury composé de météorologistes et d'enseignants. La démarche scientifique, l'implication des élèves et la présentation des projets sont des critères majeurs d'appréciation

\section{DOTATIONS}

$1^{\mathrm{er}}$ prix : $700 €-2^{\mathrm{e}}$ prix : $500 €-3^{\mathrm{e}}$ prix : $300 €$

\section{COMMENT CONCOURIR AU PRIX 2022?}

Il suffit d'envoyer avant le $\mathbf{2 0}$ mai $\mathbf{2 0 2 2}$ par e-mail à info@meteoetclimat.fr :

- Une lettre de candidature mentionnant les coordonnées postales de l'établissement,

l'adresse e-mail et le numéro de téléphone de l'enseignant responsable du projet, le niveau

de la classe participant au concours.

- L'intitulé du projet accompagné d'un descriptif détaillé (une page A4 maximum).

- Tous les supports permettant au jury d'apprécier le projet : dossier illustré, présentation

ppt, vidéos, liens vers un site web...

\section{ATTRIBUTION DES PRIX}

La décision d'attribution des prix sera communiquée avant la fin de l'année scolaire et le $1^{\text {er }}$ prix sera remis dans l'établissement lauréat en juin 2022.

Concours organisé par l'association Météo et Climat. Avec le soutien de Météo-France + d'infos : Tél. : 0149571879 - info@meteoetclimat.fr - www.meteoetclimat.fr 